\title{
ON A STOCHASTIC BURGERS EQUATION WITH DIRICHLET BOUNDARY CONDITIONS
}

\author{
EKATERINA T. KOLKOVSKA
}

Received 5 November 2002

We consider the one-dimensional Burgers equation perturbed by a white noise term with Dirichlet boundary conditions and a non-Lipschitz coefficient. We obtain existence of a weak solution proving tightness for a sequence of polygonal approximations for the equation and solving a martingale problem for the weak limit.

2000 Mathematics Subject Classification: 60H15, 60H40, 35Q35.

1. Introduction. We consider the stochastic partial differential equation

$$
\begin{aligned}
\frac{\partial}{\partial t} u(t, x)= & \frac{\partial^{2}}{\partial x^{2}} u(t, x)+f(t, x, u(t, x))+\frac{\partial}{\partial x} g(t, x, u(t, x)) \\
& +\sigma(t, x, u(t, x)) \frac{\partial^{2}}{\partial t \partial x} W(t, x)
\end{aligned}
$$

with Dirichlet boundary conditions

$$
u(t, 0)=u(t, 1)=0, \quad t \geq 0,
$$

and initial condition

$$
u(0, x)=u_{0}(x), \quad x \in[0,1]
$$

where $\left(\partial^{2} / \partial t \partial x\right) W(t, x)$ is a spacetime white noise (see [19] for the definition and properties of the white noise), $u_{0} \in L^{2}([0,1])$, and $f=f(t, x, y), g=$ $g(t, x, y)$, and $\sigma=\sigma(t, x, y)$ are Borel-measurable functions on $\mathbb{R}_{+} \times[0,1] \times \mathbb{R}$. A solution to this equation is an $L^{2}([0,1])$-valued continuous process, adapted to the filtration generated by the white noise, which solves the equation in a weak sense (see below).

When $f=\sigma=0$ and $g(t, x, y)=y^{2} / 2$, the above equation is called Burgers equation. It has been proposed as a model for turbulent fluid motion (see $[4,5,10])$. When $g=0$, the equation is a stochastic reaction-diffusion equation which has been studied intensively (see, e.g., [3, 8, 13, 19] and the references therein). 
When $f=0, g(t, x, y)=y^{2} / 2$, and $\sigma \neq 0$, we obtain the Burgers equation perturbed by a spacetime white noise. It has been studied by several authors under Lipschitz coefficients on $\sigma$ (see, e.g., [1, 2, 7, 9] and the references therein).

Our aim in this paper is to study a one-dimensional Burgers equation perturbed by a stochastic noise term with a non-Lipschitz coefficient, namely,

$$
\begin{aligned}
\frac{\partial}{\partial t} u(t, x)= & \Delta u(t, x)+\lambda \nabla u^{2}(t, x) \\
& +\gamma \sqrt{u(t, x)(1-u(t, x))} \frac{\partial^{2}}{\partial t \partial x} W(t, x), \\
u(t, 0)= & u(t, 1)=0, \\
u(0, x)= & f(x), \quad x \in[0,1]
\end{aligned}
$$

where $\Delta=\partial^{2} / \partial x^{2}, \nabla=\partial / \partial x$, and $f(x):[0,1] \rightarrow[0,1]$ is a continuous function. The stochastic term in this equation corresponds to continuous-time steppingstone models in population genetics (see $[6,15]$ ), where $u(t, x)$ models the gene frequency in colonies.

Equation (1.4) is interpreted in the weak sense, which means that, for each $\varphi \in C^{2}([0,1])$,

$$
\begin{aligned}
\int_{[0,1]} u(t, x) \varphi(x) d x= & \int_{[0,1]} u(0, x) \varphi(x) d x+\int_{[0,1]} u(t, x) \varphi^{\prime \prime}(x) d x \\
& -\lambda \int_{0}^{t} \int_{[0,1]} u^{2}(s, x) \varphi^{\prime}(x) d x d s \\
& +\gamma \int_{0}^{t} \int_{[0,1]} \sqrt{u(s, x)(1-u(s, x))} \varphi(x) W(d s, d x) .
\end{aligned}
$$

Since the coefficients of (1.4) are non-Lipschitz, the standard results on existence and uniqueness of solutions cannot be applied.

In this paper, we prove existence of a nonnegative weak solution of (1.4) (Theorem 4.2). Our method of proof is briefly described as follows. Following Funaki [8], in Section 2 we define a discrete version of (1.4), which is a finitedimensional system (2.4) of stochastic differential equations. Next, we prove existence of a weak solution for this system and use the method of Le Gall [14] to obtain pathwise uniqueness of weak solutions. This yields existence of a unique strong solution $x_{k}^{N}(t)$ of (2.4). In Section 3, we define a system of polygonal approximations $u_{N}(t, x)$ of $x_{k}^{N}(t)$ and use the multidimensional Kolmogorov-Totoki criterion to obtain tightness of $\left\{u_{N}(t, x), N=1,2, \ldots\right\}$. In Section 4 , we use a martingale problem to conclude the proof of existence of a weak solution of (1.4). Without loss of generality, we will assume that $\lambda=\gamma=1$. 
2. Existence of a solution of the discretized version. We fix an integer $N \geq$ 1 and consider the discretized version of (1.4) on the set $\{k / N, 1 \leq k \leq N\}$ :

$$
\begin{aligned}
\frac{\partial}{\partial t} X_{N}\left(t, \frac{k}{N}\right)= & \Delta_{N} X_{N}\left(t, \frac{k}{N}\right)+\nabla_{N}\left(X_{N}^{2}\left(t, \frac{k}{N}\right)\right) \\
& +\sqrt{N X_{N}\left(t, \frac{k}{N}\right)\left(1-X_{N}\left(t, \frac{k}{N}\right)\right)} d B_{k}(t), \\
X_{N}\left(0, \frac{k}{N}\right)= & f\left(\frac{k}{N}\right), \quad 1 \leq k \leq N, t \geq 0 .
\end{aligned}
$$

Here, $\mathbb{N}$ is the set of the nonnegative integer numbers, $\left\{B_{k}(t)\right\}_{1 \leq k \leq N}$ is an infinite system of independent one-dimensional Brownian motions, and $\nabla_{N}$ and $\Delta_{N}$ are, respectively, the discrete approximations of the first and second derivative with respect to the variable $x$ :

$$
\begin{aligned}
\Delta_{N} X_{N}\left(t, \frac{k}{N}\right) & =\frac{X_{N}(t,(k+1) / N)-2 X_{N}(t, k / N)+X_{N}(t,(k-1) / N)}{1 / N^{2}}, \\
\nabla_{N} h\left(s, \frac{k}{N}\right) & =\frac{h(s,(k+1) / N)-h(s, k / N)}{1 / N}, \quad 1 \leq k \leq N .
\end{aligned}
$$

We write $x_{k}^{N}(t)=X_{N}(t, k / N)$. Substituting the above expressions in (2.1), we obtain the finite-dimensional system of stochastic differential equations

$$
\begin{aligned}
d x_{i}^{N}(t)= & N^{2}\left[x_{i+1}^{N}(t)-2 x_{i}^{N}(t)+x_{i-1}^{N}(t)\right]+N x_{i+1}^{N}(t)^{2}-N x_{i}^{N}(t)^{2} \\
& +\sqrt{N x_{i}^{N}(t)\left(1-x_{i}^{N}(t)\right)} d B_{i}(t), \quad i=1, \ldots, N,
\end{aligned}
$$

which can be written in the more compact form

$$
\begin{aligned}
d x_{i}^{N}(t) & =\left(\sum_{j=1}^{N} a_{i j}^{N} x_{j}^{N}(t)+b_{i j}^{N} x_{j}^{N}(t)^{2}\right) d t+\sqrt{N x_{i}^{N}(t)\left(1-x_{i}^{N}(t)\right)} d B_{i}(t), \\
x_{i}^{N}(0) & =f\left(\frac{i}{N}\right), \quad 1 \leq i, j \leq N,
\end{aligned}
$$

where

$$
\begin{aligned}
& a_{i j}^{N}= \begin{cases}N^{2} & \text { if } j=i+1, i-1, \\
-2 N^{2} & \text { if } j=i, \\
0 & \text { otherwise, }\end{cases} \\
& b_{i j}^{N}= \begin{cases}N & \text { if } j=i+1, \\
-N & \text { if } j=i, \\
0 & \text { otherwise. }\end{cases}
\end{aligned}
$$


Note that for this system we cannot apply the standard results on existence and uniqueness of solution because Lipschitz assumptions on the drift and diffusion coefficients fail. We prove the following result.

THEOREM 2.1. For any initial random condition $X^{N}(0)=\left(x_{1}^{N}, \ldots, x_{N}^{N}\right) \in$ $[0,1]^{N}$, the system

$$
\begin{aligned}
d x_{i}^{N}(t)= & \left(\sum_{j} a_{i j}^{N} x_{j}^{N}(t)+\sum_{j} b_{i j}^{N} x_{j}^{N}(t)^{2}\right) d t \\
& +\sqrt{N x_{i}^{N}(t)\left(1-x_{i}^{N}(t)\right)} d B_{i}(t), \\
x_{i}^{N}(0)= & x_{i}, \quad i=1, \ldots, N,
\end{aligned}
$$

admits a unique strong solution $X^{N}(t)=\left(x_{1}^{N}(t), \ldots, x_{N}^{N}(t)\right) \in C\left([0, \infty),[0,1]^{N}\right)$.

Proof. We consider the rescaled system

$$
\begin{aligned}
d x_{i}^{N}(t)= & \left(\sum_{j} a_{i j}^{N} x_{j}^{N}(t)+\sum_{j} b_{i j}^{N} x_{j}^{N}(t)^{2}\right) d t \\
& +\sqrt{g\left(x_{i}^{N}(t)\right)} d B_{i}(t), \\
x_{i}^{N}(0)= & x_{i}, \quad i=1, \ldots, N,
\end{aligned}
$$

where $g: \mathbb{R} \rightarrow \mathbb{R}$ is defined by $g(x)=N x(1-x)$ for $0 \leq x \leq 1$, and $g(x)=0$ otherwise. Since the coefficients of (2.7) are continuous, by the Skorokhod's existence theorem (see $[11,17]$ ), we conclude that there exists on some probability space a weak solution $X^{N}(t)$ of (2.7). We will prove that for each weak solution $X^{N}(t)=\left(x_{1}^{N}(t), \ldots, x_{N}^{N}(t)\right)$ of this system, $x_{i}^{N}(t) \in[0,1]$ for all $i=1, \ldots, N$ and $t \geq 0$, thus showing that $X^{N}(t)$ is a solution of (2.6).

First, we show that $x_{i}^{N}(t) \geq 0$ for each $i=1, \ldots, N$. Since the coefficients of the system are non-Lipschitz, the solution may explode in a finite time. Let $\tau_{1} \leq \infty$ denote the explosion time of the solution. If some of the solution coordinates are negative, then there exists a random time $0<\tau_{2} \leq \infty$ such that for $0<t \leq \tau_{2}$, all such coordinates are between -1 and 0 . This is so because (2.7) is finite-dimensional, and its solution is continuous.

We will use the following lemma (see [14]).

LEMMA 2.2. Let $Z \equiv\{Z(t), t \geq 0\}$ be a real-valued semimartingale. Suppose that there exists a function $\rho:[0, \infty) \rightarrow[0, \infty)$ such that $\int_{0}^{\varepsilon} d u / \rho(u)=+\infty$ for all $\varepsilon>0$, and $\int_{0}^{t}\left(1_{\left\{Z_{s}>0\right\}} / \rho\left(Z_{s}\right)\right) d\langle Z\rangle_{s}<\infty$ for all $t>0$ a.s. Then the local time at zero of $Z, L_{t}^{0}(Z)$, is identically zero for all $t$ a.s. 
Applying Lemma 2.2 to $x_{i}^{N}(t)$ with $\rho(u)=u$ and using the Tanaka formula (see [16]), we obtain for $x_{i}^{N}(t)_{-}:=\max \left[0,-x_{i}^{N}(t)\right]$,

$$
\begin{aligned}
\sum_{i=1}^{N} x_{i}^{N}(t)_{-} & =-\int_{0}^{t} \sum_{i=1}^{N} 1_{x_{i}^{N}(s)<0} \sum_{j=1}^{N}\left(a_{i j}^{N} x_{j}(s)+b_{i j}^{N} x_{j}(s)^{2}\right) d s \\
& \leq \int_{0}^{t} \sum_{i, j=1}^{N} 1_{x_{i}^{N}(s)<0} a_{i j}^{N} x_{j}(s)_{-} d s+N \int_{0}^{t} \sum_{i=1}^{N} 1_{x_{i}^{N}(s)<0} x_{i}(s)^{2} d s \\
& \leq \int_{0}^{t} \sum_{i, j=1}^{N} a_{i j}^{N} x_{j}(s)_{-} d s+N \int_{0}^{t} \sum_{i=1}^{N} x_{i}^{N}(s)_{-} d s \\
& =N \int_{0}^{t} \sum_{i=1}^{N} x_{i}^{N}(s)_{-} d s,
\end{aligned}
$$

where we used that $\sum_{i} a_{i j}^{N}=0$ to obtain the last equality. Then by Gronwall's lemma, we obtain that $\sum_{i=1}^{N} x_{i}^{N}(t)_{-}=0$, and hence that the solution is nonnegative for each $t \geq 0$. By a similar argument applied to $\left(1-x_{i}^{N}(t)\right)_{-}$, it follows that $x_{i}^{N}(t) \leq 1$ for each $1 \leq i \leq N$.

Using Lemma 2.2, we will prove pathwise uniqueness of weak solutions of (2.6). Let $X^{1, N}=\left(x_{1}^{1, N}, \ldots, x_{N}^{1, N}\right)$ and $X^{2, N}=\left(x_{1}^{2, N}, \ldots, x_{N}^{2, N}\right)$ be two solutions of (2.6) with the same initial conditions and the same Brownian motions. We define

$$
d_{i}\left(X^{l, N}(t)\right)=a_{i j}^{N} x_{j}^{l, N}(t)+b_{i j}^{N} x_{j}^{l, N}(t)^{2}, \quad t \geq 0, l=1,2
$$

Then

$$
\begin{aligned}
x_{i}^{1, N}(t)-x_{i}^{2, N}(t)= & \int_{0}^{t}\left[d_{i}\left(X^{1, N}(s)\right)-d_{i}\left(X^{2, N}(s)\right)\right] d s \\
& +\int_{0}^{t}\left[\sqrt{N x_{i}^{1, N}(s)\left(1-x_{i}^{1, N}(s)\right)}\right. \\
& \left.\quad-\sqrt{N x_{i}^{2, N}(s)\left(1-x_{i}^{2, N}\right)(s)}\right] d B_{i}(s), \quad i=1, \ldots, N .
\end{aligned}
$$

Since

$$
\begin{gathered}
\langle X\rangle_{t}=\int_{0}^{t}\left[\sqrt{N x_{i}^{1, N}(s)\left(1-x_{i}^{1, N}(s)\right)}-\sqrt{N x_{i}^{2, N}(s)\left(1-x_{i}^{2, N}\right)}(s)\right]^{2} d s, \\
\int_{0}^{t} \frac{\left[\sqrt{N x_{i}^{1, N}(s)\left(1-x_{i}^{1, N}(s)\right)}-\sqrt{N x_{i}^{2, N}(s)\left(1-x_{i}^{2, N}\right)(s)}\right]^{2}}{x_{i}^{1, N}(s)-x_{i}^{2, N}(s)} 1_{x_{i}^{1, N}(s)-x_{i}^{2, N}(s)>0} d s \\
\leq \int_{0}^{t} 2 N 1_{x_{i}^{1, N}(s)-x_{i}^{2, N}(s)>0} d s<2 N t,
\end{gathered}
$$


(where we used that $(\sqrt{x(1-x)}-\sqrt{y(1-y)}) /(x-y)<2$ for $x, y \in[0,1]$, $x>y$, which follows from L'Hospital rule), we can apply Lemma 2.2 to $X(t)=$ $x_{i}^{1, N}(t)-x_{i}^{2, N}(t)$ with $\rho(x)=x$. Therefore, $L_{t}^{0}\left(x_{i}^{1, N}(s)-x_{i}^{2, N}(s)\right)=0$ for all $i \in\{1, \ldots, N\}$.

By Tanaka's formula,

$$
\begin{aligned}
\left|x_{i}^{1, N}(t)-x_{i}^{2, N}(t)\right|= & \int_{0}^{t} \operatorname{sgn}\left(x_{i}^{1, N}(s)-x_{i}^{2, N}(s)\right)\left(d_{i}\left(X^{1, N}(s)\right)-d_{i}\left(X^{2, N}(s)\right)\right) d s \\
+ & \int_{0}^{t} \operatorname{sgn}\left(x_{i}^{1, N}(s)-x_{i}^{2, N}(s)\right) \\
\times & {\left[\sqrt{N x_{i}^{1, N}(s)\left(1-x_{i}^{1, N}(s)\right)}\right.} \\
& \left.-\sqrt{N x_{i}^{2, N}(s)\left(1-x_{i}^{2, N}(s)\right)}\right] d B_{i}(s), \quad i=1, \ldots, N .
\end{aligned}
$$

Since $a_{i j}^{N}$ and $b_{i j}^{N}$ are bounded, it follows that

$$
\begin{aligned}
\mathbb{E} \sum_{i=1}^{N}\left|x_{i}^{1, N}(t)-x_{i}^{2, N}(t)\right| & \leq \int_{0}^{t} \mathbb{E} \sum_{i=1}^{N}\left|d_{i}\left(X^{1, N}(s)\right)-d_{i}\left(X^{2, N}(s)\right)\right| d s \\
& \leq \int_{0}^{t} K(N) \mathbb{E} \sum_{i=1}^{N}\left|x_{i}^{1, N}(s)-x_{i}^{2, N}(s)\right| d s,
\end{aligned}
$$

where $K(N)$ is a constant depending on $N$. From Gronwall's inequality, we conclude that

$$
\mathbb{E} \sum_{i=1}^{d}\left|x_{i}^{1, N}(t)-x_{i}^{2, N}(t)\right|=0
$$

for all $t \geq 0$, thus proving pathwise uniqueness. By a classical theorem of Yamada and Watanabe [20], this is sufficient for existence of a unique strong solution of (2.6).

3. Tightness of the approximating processes. From Theorem 2.1, there exists a strong solution of the system approximating (1.4)

$$
\begin{aligned}
d x_{i}^{N}(t) & =\sum_{1 \leq j \leq N} a_{i j}^{N} x_{j}^{N}(t)+\sum_{1 \leq j \leq N} b_{i j}^{N} x_{j}^{N}(t)^{2}+\sqrt{N x_{i}^{N}(t)\left(1-x_{i}^{N}(t)\right)} d B_{i}(t), \\
x_{i}^{N}(0) & =f\left(\frac{i}{N}\right), \quad i=1,2, \ldots, N
\end{aligned}
$$


where $N \in \mathbb{N}$ is fixed. We denote by $u_{N}(t, x)$ the polygonal approximation of $x_{i}^{N}(t)$

$$
\begin{aligned}
u_{N}(t, x)= & X_{N}\left(t, \frac{[N x]+1}{N}\right)(N x-[N x]) \\
& +X_{N}\left(t, \frac{[N x]}{N}\right)([N x]+1-N x), \quad t \geq 0, x \in[0,1],
\end{aligned}
$$

where by definition, $[y]=k / N$ for $k / N \leq y<(k+1) / N$. Therefore, we have $X_{N}(t, k / N)=x_{k}^{N}(t)=u_{N}(t, k / N), 1 \leq k \leq N$, and $0 \leq u_{N}(t, x) \leq 1$ for all $t \geq 0$, $x \in[0,1]$.

Let $p_{N}(t, i / N, j / N), t \geq 0,0 \leq i, j \leq N+1$, be the fundamental solution of $\Delta_{N}$, that is,

$$
\begin{aligned}
\frac{\partial}{\partial t} p_{N}\left(t, \frac{i}{N}, \frac{j}{N}\right) & =\Delta_{N} p_{N}\left(t, \frac{i}{N}, \frac{j}{N}\right), \quad t>0,1 \leq i, j \leq N, \\
p_{N}\left(0, \frac{i}{N}, \frac{j}{N}\right) & =N \delta_{i j},
\end{aligned}
$$

with the boundary conditions

$$
p_{N}\left(t, 0, \frac{j}{N}\right)=p_{N}\left(t, \frac{N+1}{N}, \frac{j}{N}\right)=0, \quad t>0,1 \leq j \leq N
$$

Then (3.1) is equivalent to the system (see [12])

$$
\begin{aligned}
x_{i}^{N}(t)= & \sum_{j=1}^{N} \frac{1}{N} p_{N}\left(t, \frac{i}{N}, \frac{j}{N}\right) x_{j}^{N}(0) \\
& +\int_{0}^{t}\left[\sum_{j=1}^{N} \frac{1}{N} p_{N}\left(t-s, \frac{i}{N}, \frac{j}{N}\right) b(i, j) x_{j}^{N}(s)^{2}\right] d s \\
& +\int_{0}^{t} \sum_{j=1}^{N}\left[p_{N}\left(t-s, \frac{i}{N}, \frac{j}{N}\right) \sqrt{N x_{j}^{N}(s)\left(1-x_{j}^{N}(s)\right)}\right] d B_{j}(s), \quad 1 \leq i \leq N,
\end{aligned}
$$

where in the last integral we used that $\left\{(1 / N) B_{j}(s), 1 \leq j \leq N\right\}$ is an independent system of Brownian motions which we also denote by $\left\{B_{j}(s)\right\}$.

We define the rescaled polygonal interpolation $G_{N}$ of $p_{N}$ in $[0,1]$ by

$$
\begin{aligned}
G_{N}\left(t, x, \frac{j}{N}\right)= & p_{N}\left(t, \frac{[N x]+1}{N}, \frac{j}{N}\right)(N x-[N x]) \\
& +p_{N}\left(t, \frac{[N x]}{N}, \frac{j}{N}\right)([N x]+1-N x) .
\end{aligned}
$$


Using (3.2) and (3.8), we obtain the following representation for the approximate solution. For $x \in[i / N,(i+1) / N)$,

$$
\begin{aligned}
u_{N}(t, x)= & \sum_{j=1}^{N} \frac{1}{N} G_{N}\left(t, x, \frac{j}{N}\right) u_{N}\left(0, \frac{j}{N}\right) \\
& +\int_{0}^{t} \sum_{j=1}^{N}\left[\frac{1}{N} p_{N}\left(t-s, \frac{i+1}{N}, \frac{j}{N}\right) b(i+1, j) x_{j}^{N}(s)^{2}(N x-[N x])\right. \\
& \left.\quad+\frac{1}{N} p_{N}\left(t-s, \frac{i}{N}, \frac{j}{N}\right) b(i, j) x_{j}^{N}(s)^{2}([N x]+1-N x)\right] d s \\
+ & \int_{0}^{t} \sum_{j=1}^{N} G_{N}\left(t-s, x, \frac{j}{N}\right) \sqrt{N u_{N}\left(s, \frac{j}{N}\right)\left(1-u_{N}\left(s, \frac{j}{N}\right)\right)} d B_{j}(s) d s \\
:= & u_{N}^{(1)}(t, x)+u_{N}^{(2)}(t, x)+u_{N}^{(3)}(t, x) .
\end{aligned}
$$

Then $u_{N}(t, x)$ satisfies the boundary conditions in (1.4).

THEOREM 3.1. For each $T>0$, the sequence $\left\{u_{N}(t, x), N \geq 1\right\}$ is tight in the space $C([0, T], A)$, where $A=C([0,1],[0,1])$.

Proof. Using the fact that $u_{N}(t, x) \in[0,1]$, we obtain, as in the proofs of [8, Lemma 2.2] and [8, Proposition 2.1], that for each $T<\infty$ and $p \in \mathbb{N}$, there exists $C=C(T, p)>0$ such that

$$
E\left|u_{N}^{(3)}\left(t_{1}, x\right)-u_{N}^{(3)}\left(t_{2}, y\right)\right|^{2 p} \leq C\left(\left|t_{1}-t_{2}\right|^{p / 2}+|x-y|^{p / 2}\right)
$$

for every $t_{1}, t_{2} \in[0, T], x, y \in[0,1]$, and $N \in \mathbb{N}$, and that

$$
\lim _{N \rightarrow \infty} \sup _{(t, y) \in[0, T] \times[0,1]}\left|u_{N}^{(1)}(t, y)-u(t, y)\right|=0
$$

where $u(t, y)$ is the fundamental solution of $\Delta$. Since

$$
u_{N}^{(2)}\left(t, \frac{k}{N}\right)=\int_{0}^{t}\left[p_{N}\left(t-s, \frac{k}{N}, \frac{k+1}{N}\right) x_{k+1}^{N}(s)^{2}-p_{N}\left(t-s, \frac{k}{N}, \frac{k}{N}\right) x_{k}^{N}(s)^{2}\right] d s
$$

and $p_{N}$ is a fundamental solution of $\Delta_{N}$, it follows that

$$
\frac{\partial}{\partial t} u_{N}^{(2)}\left(t, \frac{k}{N}\right)=\Delta_{N} u_{N}^{(2)}\left(t, \frac{k}{N}\right)+u_{N}\left(t, \frac{k+1}{N}\right)^{2}-u_{N}\left(t, \frac{k}{N}\right)^{2} .
$$


Also, $u_{N}^{(2)}(t, 0)=u_{N}^{(2)}(t, 1)=0$ by the boundary condition (3.8). Integration by parts and an application of Gronwall's inequality give, as in [12, Theorem 4.2],

$$
\max _{1 \leq k \leq N}\left|u_{N}^{(2)}\left(t, \frac{k}{N}\right)\right| \leq e^{2 t} \int_{0}^{t} \max _{1 \leq k \leq N}\left|u_{N}^{(1)}\left(s, \frac{k}{N}\right)+u_{N}^{(3)}\left(s, \frac{k}{N}\right)\right| d s .
$$

Hence, from the polygonal form of $u_{N}^{(2)}$ and (3.8), (3.9), we obtain that for every $T<\infty$ and $p \in \mathbb{N}$, there exists $C_{1}=C_{1}(T, p)>0$ such that

$$
E\left|u_{N}^{(2)}\left(t_{1}, x\right)-u_{N}^{(2)}\left(t_{2}, y\right)\right|^{2 p} \leq C_{1}\left(\left|t_{1}-t_{2}\right|^{p / 2}+|x-y|^{p / 2}\right)
$$

for every $t_{1}, t_{2} \in[0, T], x, y \in[0,1]$, and $N \in \mathbb{N}$. It follows from the multidimensional Kolmogorov-Totoki criterion [18] that $u_{N}(t, x) \in C([0, T], A)$ and that the family $\left\{u_{N}(t, x), N \in \mathbb{N}\right\}$ is tight in $C([0, T], A)$ for each positive $T$.

\section{The martingale problem for the stochastic partial differential}

equation. Since the sequence $\left\{u_{N}(t, x), N \geq 1\right\}$ is tight by Theorem 3.1, there exists a subsequence, which we denote again by $\left\{u_{N_{k}}(t, x)\right\}$, that converges weakly in $C([0, T], A)$ to a limit $v(t, x)$. By the Skorokhod's representation theorem, we can construct processes $\left\{v_{N}(t, x)\right\}, u(t, x)$ on some probability space $\left(\Omega, \mathscr{F}_{F},\left\{\mathscr{F}_{t}\right\}, P\right)$ such that $\left\{u_{N}\right\} \stackrel{\mathscr{P}}{=}\left\{v_{N}\right\}, u \stackrel{\mathscr{D}}{=} v$, and $\left\{v_{N}(t, x)\right\}$ converges to $u(t, x)$ uniformly on compact subsets of $[0, T] \times \mathbb{R}$ for any $T>0$ as $N \rightarrow \infty$. Obviously, $u(t, x)$ satisfies the boundary conditions in (1.4). We will show that $u(t, x)$ is a weak solution of (1.4) by solving the corresponding martingale problem.

THEOREM 4.1. For any $\varphi \in C_{c}^{2}$,

$$
\begin{aligned}
\mu_{\varphi}(t):= & \int_{[0,1]} u(t, x) \varphi(x) d x-\int_{[0,1]} u(0, x) \varphi(x) d x \\
& -\int_{[0,1]} u(t, x) \varphi^{\prime \prime}(x) d x-\int_{0}^{t} \int_{[0,1]} u^{2}(s, x) \varphi^{\prime}(x) d x
\end{aligned}
$$

is an $\left\{\mathscr{F}_{t}\right\}$-martingale with $\left\langle\mu_{\varphi}\right\rangle_{t}=\int_{0}^{t} \int_{[0,1]} u(s, x)(1-u(s, x)) \varphi^{2}(x) d x d s$.

Proof. Using that

$$
\sum_{n \in \mathbb{Z}} a_{n}\left(b_{n+1}-b_{n}\right)+\sum_{n \in \mathbb{Z}} b_{n+1}\left(a_{n+1}-a_{n}\right)=0
$$


and multiplying both sides of (2.1) by $\varphi(k / N)(1 / N)$, we obtain, for fixed $N \geq 1$,

$$
\begin{aligned}
\mu_{\varphi}^{N}(t):= & \sum_{k=1}^{N} u_{N}\left(t, \frac{k}{N}\right) \varphi\left(\frac{k}{N}\right) \frac{1}{N}-\sum_{k=1}^{N} u_{N}\left(0, \frac{k}{N}\right) \varphi\left(\frac{k}{N}\right) \frac{1}{N} \\
& -\int_{0}^{t} \sum_{k=1}^{N} \Delta_{N} u_{N}\left(s, \frac{k}{N}\right) \varphi\left(\frac{k}{N}\right) \frac{1}{N}-\int_{0}^{t} \sum_{k=1}^{N} \nabla_{N}\left\{u_{N}^{2}\left(s, \frac{k}{N}\right)\right\} \varphi\left(\frac{k}{N}\right) \frac{1}{N} \\
= & \sum_{k} v_{N}\left(t, \frac{k}{N}\right) \varphi\left(\frac{k}{N}\right) \frac{1}{N}-\sum_{k} v_{N}\left(0, \frac{k}{N}\right) \varphi\left(\frac{k}{N}\right) \frac{1}{N} \\
& -\int_{0}^{t} \sum_{k} v_{N}\left(s, \frac{k}{N}\right) \Delta_{N} \varphi\left(\frac{k}{N}\right) \frac{1}{N}-\int_{0}^{t} \sum_{k} v_{N}^{2}\left(s, \frac{k}{N}\right) \nabla_{N} \varphi\left(\frac{k}{N}\right) \frac{1}{N} \\
= & \sum_{k} \varphi\left(\frac{k}{N}\right) \frac{1}{N} \int_{0}^{t} \sqrt{N v_{N}\left(s, \frac{k}{N}\right)\left(1-v_{N}\left(s, \frac{k}{N}\right)\right)} d B_{k}(s) .
\end{aligned}
$$

Hence, $\mu_{\varphi}^{N}(t)$ is a martingale because by $(4.3), \mathcal{M}_{\varphi}^{N}(t)$ is the sum of a finite number of martingales. Moreover, $\left\{\mathcal{M}_{\varphi}^{N}(t)\right\}$ is uniformly integrable because $\sup _{N \in \mathbb{N}} \mathbb{E}\left(M_{\varphi}^{N}(t)\right)^{2}<\infty$ uniformly in $t \in[0, T]$. Indeed, since $\varphi^{2}$ is integrable,

$$
\begin{aligned}
\mathbb{E}\left(\mathcal{M}_{\varphi}^{N}(t)\right)^{2} & =\sum_{k} \varphi^{2}\left(\frac{k}{N}\right) \frac{1}{N} \int_{0}^{t}\left[v_{N}\left(s, \frac{k}{N}\right)\left(1-v_{N}\left(s, \frac{k}{N}\right)\right)\right] d s \\
& \leq T \sum_{k} \frac{1}{N} \varphi^{2}\left(\frac{k}{N}\right) \\
& <C(\varphi, T),
\end{aligned}
$$

where $C(\varphi, T)$ is a finite constant depending only on $\varphi$ and $T$, but not on $N$. Therefore, $\mu_{\varphi}^{N}(t) \rightarrow \mu_{\varphi}(t)$ as $N \rightarrow \infty$, where

$$
\begin{aligned}
\mu_{\varphi}(t)= & \int_{[0,1]} v(t, x) \varphi(x) d x-\int_{[0,1]} v(t, 0) \varphi(x) d x \\
& -\int_{0}^{t} \int_{0}^{1} v(s, x) \varphi^{\prime \prime}(x) d x d s-\int_{0}^{t} \int_{0}^{1} v^{2}(s, x) \varphi^{\prime}(x) d x d s
\end{aligned}
$$

is a martingale. From (4.3), we obtain the quadratic variation of $\mu_{\varphi}(t)$, which is given by

$$
\begin{aligned}
\left\langle\mu_{N}(\varphi)\right\rangle_{t} & =\left\langle\sum_{k} \varphi\left(\frac{k}{N}\right) \frac{1}{N} \int_{0}^{t} \sqrt{N v_{N}\left(s, \frac{k}{N}\right)\left(1-v_{N}\left(s, \frac{k}{N}\right)\right)} d B_{k}(s)\right\rangle_{t} \\
& =\int_{0}^{t} \sum_{k} N v_{N}\left(s, \frac{k}{N}\right)\left(1-v_{N}\left(s, \frac{k}{N}\right)\right) \varphi^{2}\left(\frac{k}{N}\right) \frac{1}{N^{2}} d s .
\end{aligned}
$$


Hence, $\lim _{N \rightarrow \infty}\left\langle\mathcal{M}_{N}(\varphi)\right\rangle_{t}=\int_{0}^{t} \int_{[0,1]} v(s, x)(1-v(s, x)) \varphi^{2}(x) d x d s=\langle\mathcal{M}(\varphi)\rangle_{t}$, and the theorem is proved.

Now, we proceed to the proof of the main result.

THEOREM 4.2. The process $u(t, x)$ is a weak solution of the stochastic partial differential equation (1.4).

Proof. To the quadratic variation $\langle\mathcal{M}(\varphi)\rangle_{t}$, there corresponds a martingale measure $\mathcal{M}(d t, d x)$ with quadratic measure

$$
v(d x d t)=u(t, x)(1-u(t, x)) d x d t
$$

(see [19]). Let $\widetilde{W}$ be a white noise independent of $\mathcal{M}$ (defined possibly on an extended probability space). We define

$$
\begin{aligned}
W_{t}(\varphi)= & \int_{[0,1]} \int_{0}^{t} \frac{1}{u(s, x)(1-u(s, x))} 1_{\{u(s, x) \notin\{0,1\}\}} \varphi(x) \mu(d s, d x) \\
& +\int_{[0,1]} \int_{0}^{t} 1_{\{u(s, x) \in\{0,1\}\}} \varphi(x) \widetilde{W}(d s, d x) .
\end{aligned}
$$

Then $W_{t}$ corresponds to a spacetime white noise $W(d s, d x)$ such that

$$
\mu_{t}(\varphi)=\int_{[0,1]} \int_{0}^{t} \sqrt{u(s, x)(1-u(s, x))} \varphi(x) W(d s, d x) .
$$

From (4.5), we conclude that $u(t, x)$ satisfies (1.5), and hence that $u(t, x)$ is a weak solution of (1.4). The theorem is proved.

ACKNOWLEDGMENTS. The author wishes to acknowledge the referees for their careful reading of the manuscript and their useful comments. This research was partially supported by CONACYT Grant 32401E.

\section{REFERENCES}

[1] L. Bertini, N. Cancrini, and G. Jona-Lasinio, The stochastic Burgers equation, Comm. Math. Phys. 165 (1994), no. 2, 211-232.

[2] P. Biler, T. Funaki, and W. A. Woyczynski, Fractal Burgers equations, J. Differential Equations 148 (1998), no. 1, 9-46.

[3] D. Blount, Density-dependent limits for a nonlinear reaction-diffusion model, Ann. Probab. 22 (1994), no. 4, 2040-2070.

[4] J. M. Burgers, A mathematical model illustrating the theory of turbulence, Advances in Applied Mechanics (R. von Mises and T. von Kármán, eds.), Academic Press, New York, 1948, pp. 171-199.

[5] _ The Nonlinear Diffusion Equation. Asymptotic Solutions and Statistical Problems, D. Reidel Publishing, Dordrecht, 1974.

[6] J. F. Crow and M. Kimura, An Introduction to Population Genetics Theory, Harper \& Row Publishers, New York, 1970.

[7] G. Da Prato and D. Gatarek, Stochastic Burgers equation with correlated noise, Stochastics Stochastics Rep. 52 (1995), no. 1-2, 29-41. 
[8] T. Funaki, Random motion of strings and related stochastic evolution equations, Nagoya Math. J. 89 (1983), 129-193.

[9] I. Gyöngy, Existence and uniqueness results for semilinear stochastic partial differential equations, Stochastic Process. Appl. 73 (1998), no. 2, 271-299.

[10] E. Hopf, The partial differential equation $u_{t}+u u_{x}=\mu u_{x x}$, Comm. Pure Appl. Math. 3 (1950), 201-230.

[11] N. Ikeda and S. Watanabe, Stochastic Differential Equations and Diffusion Processes, North-Holland Mathematical Library, vol. 24, North-Holland Publishing, Amsterdam, 1989.

[12] K. Iwata, An infinite-dimensional stochastic differential equation with state space $C(\mathbf{R})$, Probab. Theory Related Fields 74 (1987), no. 1, 141-159.

[13] P. Kotelenez, High density limit theorems for nonlinear chemical reactions with diffusion, Probab. Theory Related Fields 78 (1988), no. 1, 11-37.

[14] J.-F. Le Gall, Applications du temps local aux équations différentielles stochastiques unidimensionnelles [Local time applications to one-dimensional stochastic differential equations], Seminar on Probability, XVII, Lecture Notes in Math., vol. 986, Springer, Berlin, 1983, pp. 15-31 (French).

[15] T. Maruyama, Stochastic Problems in Population Genetics, Lecture Notes in Biomathematics, vol. 17, Springer-Verlag, Berlin, 1977.

[16] D. Revuz and M. Yor, Continuous Martingales and Brownian Motion, 2nd ed., Grundlehren der Mathematischen Wissenschaften, vol. 293, SpringerVerlag, Berlin, 1994.

[17] A. V. Skorokhod, Studies in the Theory of Random Processes, Addison-Wesley Publishing, Massachusetts, 1965.

[18] H. Totoki, A method of construction of measures on function spaces and its applications to stochastic processes, Mem. Fac. Sci. Kyushu Univ. Ser. A 15 (1961/1962), 178-190.

[19] J. B. Walsh, An introduction to stochastic partial differential equations, École d'été de probabilités de Saint-Flour XIV, 1984, Lecture Notes in Math., vol. 1180, Springer, Berlin, 1986, pp. 265-439.

[20] T. Yamada and S. Watanabe, On the uniqueness of solutions of stochastic differential equations, J. Math. Kyoto Univ. 11 (1971), 155-167.

Ekaterina T. Kolkovska: Centro de Investigacion en Matematicas (CIMAT), Guanajuato, CP 36000, Mexico 


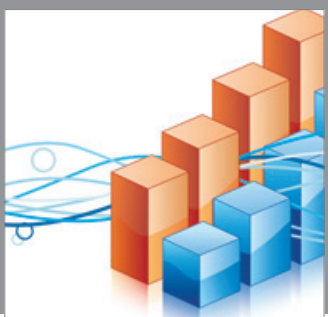

Advances in

Operations Research

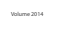

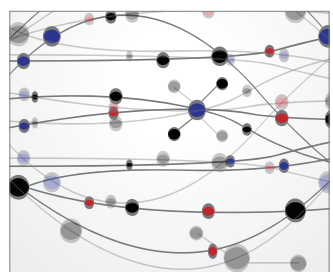

\section{The Scientific} World Journal
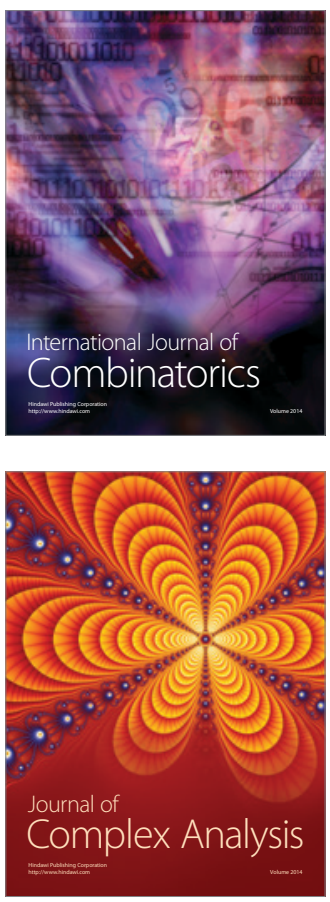

International Journal of

Mathematics and

Mathematical

Sciences
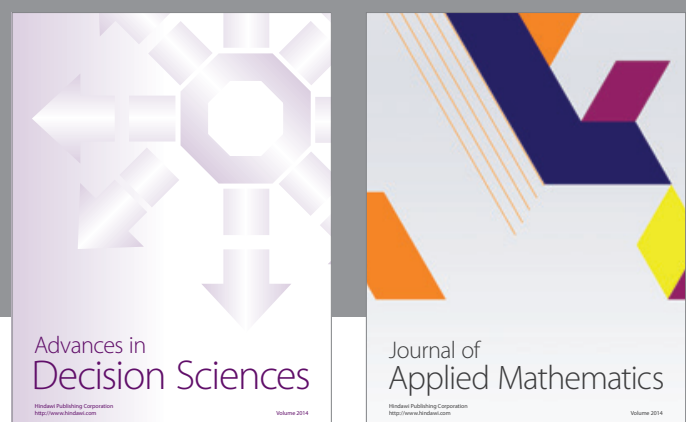

Journal of

Applied Mathematics
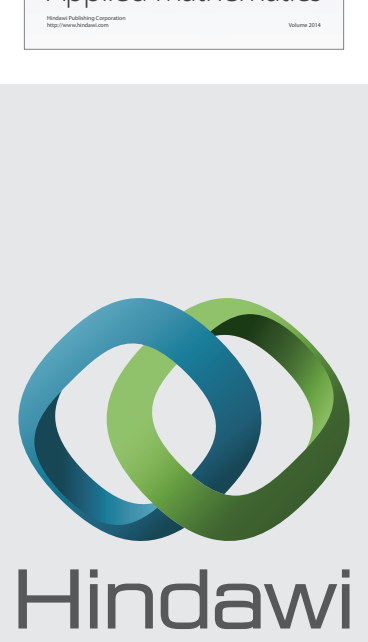

Submit your manuscripts at http://www.hindawi.com
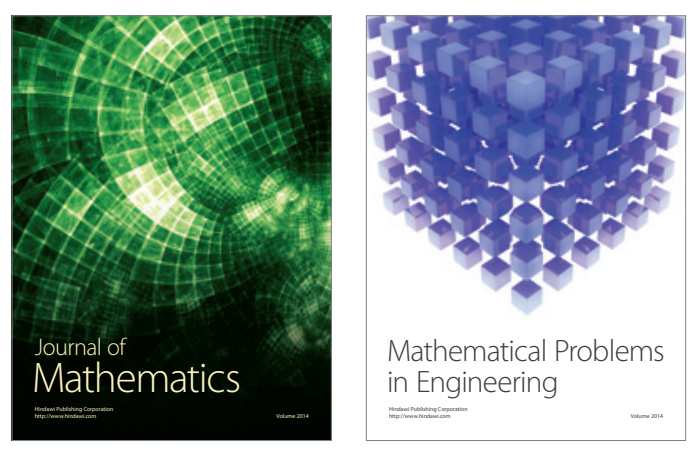

Mathematical Problems in Engineering
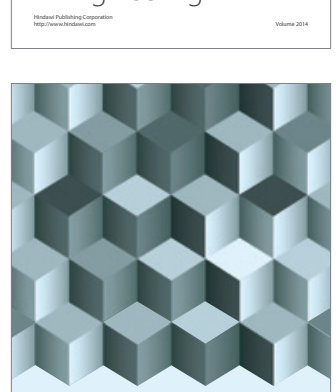

Journal of

Function Spaces
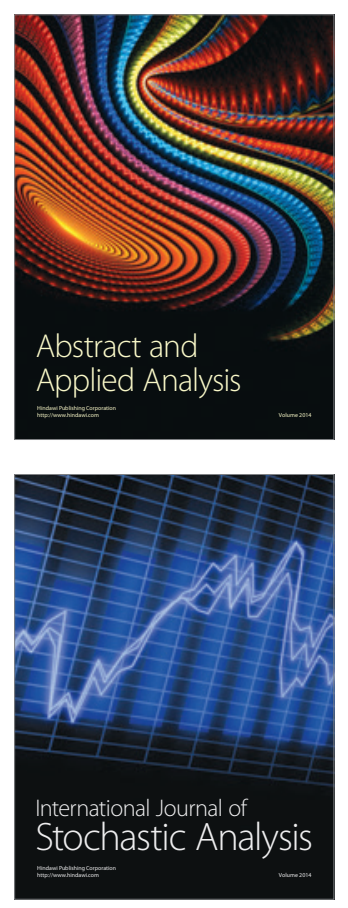

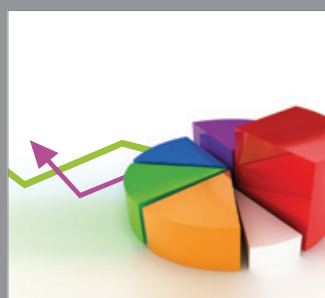

ournal of

Probability and Statistics

Promensencen
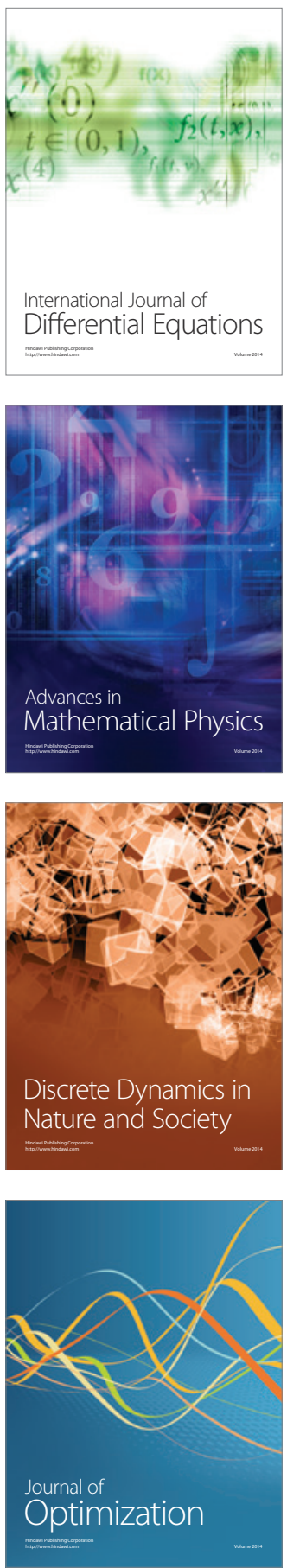